\title{
Fetal glucocorticoid receptor (Nr3c1) deficiency alters the landscape of DNA methylation of murine placenta in a sex- dependent manner and is associated to anxiety-like behavior in adulthood
}

\author{
Michaela Schmidt ${ }^{1}$, Elad Lax ${ }^{2,3}$, Rudy Zhou ${ }^{2}$, David Cheishvili, ${ }^{2,3}$, Arne Mathias Ruder ${ }^{1}$, Alessia Ludiro ${ }^{4}$, Florian Lapert ${ }^{1}$, \\ Anna Macedo da Cruz ${ }^{1}$, Paolo Sandrini ${ }^{4}$, Teresa Calzoni ${ }^{4}$, Farida Vaisheva $\mathbb{E}^{2}$, Christiane Brandwein ${ }^{1}$, Alessia Luoni ${ }^{4}$, \\ Renaud Massart ${ }^{3,5}$, Laurence Lanfumey ${ }^{5,6}$, Marco Andrea Riva $\mathbb{1}^{4}$, Michael Deuschle ${ }^{1}$, Peter Gass ${ }^{1}$ and Moshe Szyf ${ }^{2,3}$
}

\begin{abstract}
Prenatal stress defines long-term phenotypes through epigenetic programming of the offspring. These effects are potentially mediated by glucocorticoid release and by sex. We hypothesized that the glucocorticoid receptor (Gr, $\mathrm{Nr3C1)}$ fashions the DNA methylation profile of offspring. Consistent with this hypothesis, fetal Nr3C1 heterozygosity leads to altered DNA methylation landscape in fetal placenta in a sex-specific manner. There was a significant overlap of differentially methylated genes in fetal placenta and adult frontal cortex in Nr3cl heterozygotes. Phenotypically, $\mathrm{Nr3C1}$ heterozygotes show significantly more anxiety-like behavior than wildtype. DNA methylation status of fetal placental tissue is significantly correlated with anxiety-like behavior of the same animals in adulthood. Thus, placental DNA methylation might predict behavioral phenotypes in adulthood. Our data supports the hypothesis that Nr3c1 influences DNA methylation at birth and that DNA methylation in placenta correlates with adult frontal cortex DNA methylation and anxiety-like phenotypes.
\end{abstract}

\section{Introduction}

The glucocorticoid receptor (GR, NR3C1) plays a major role in the development of stress-induced disorders ${ }^{1}$. Prenatal stress exerts a strong impact on the HPA-axis of rat offspring into adulthood (e.g., ${ }^{2,3}$ ). The behavior of affected animals is altered during the whole lifespan: pups display increased ultrasonic vocalizations when stressed prenatally ${ }^{4}$, adolescent rats show less social play $^{5}$, and adult animals show more anxiety- and depressive-like

\footnotetext{
Correspondence: Michaela Schmidt (michaela.schmidt@zi-mannheim.de) ${ }^{1}$ Central Institute of Mental Health Mannheim (ZI), Medical Faculty of Mannheim, University of Heidelberg, J5, 68159 Mannheim, Germany

${ }^{2}$ Department of Pharmacology \& Therapeutics, McGill University, Montreal, QC H3G 1Y6, Canada

Full list of author information is available at the end of the article.
}

behavior ${ }^{6}$. These results have been found in humans as well $^{7}$. Moreover, there is an abundance of studies showing sex differences in stress-programming regarding behavioral, physiological, endocrine, and epigenetic modifications ${ }^{8}$.

As the stress response is mediated by glucocorticoids, they are potentially a primary programming factor conveying maternal stress to the fetus via the placenta ${ }^{9}$. This assumption is supported by the evidence that treatment with synthetic glucocorticoids of pregnant rodents leads to offspring with similar HPA-axis and behavioral changes as prenatally stressed offspring ${ }^{10}$. Moreover, when maternal adrenal glands are removed, prenatally stressed offspring do not display the full phenotype of increased stress responsivity ${ }^{11}$. There is a large variety of methods 
for applying prenatal stress in animal models (e.g., ${ }^{9,12,13}$ ). Loss of NR3C1 function in the nervous system disturbs hypothalamus-pituitary-adrenal-axis regulation so that glucocorticoid levels ${ }^{14}$ as well as stress responsivity are elevated $^{15,16}$. In this study, we use a heterozygous $N r 3 c 1$ knockout mouse to determine the role of the fetal Nr3c1 gene in defining DNA methylation patterns and future behavior.

The glucocorticoid receptor has been demonstrated to be a crucial target for epigenetic programming by early postnatal experiences: differences in maternal care in the rat stably alter the methylation state of the promotor region of the glucocorticoid receptor in the hippocampus of offspring ${ }^{17}$. Increased maternal licking/grooming caused a lower stress responsivity of offspring compared to the offspring of dams with low levels of licking/ grooming. Epigenetic pharmacological manipulation reversed the effects of maternal care on DNA methylation as well as behavior, supporting the idea that epigenetic differences mediated the effect of maternal care on the behavior of the offspring ${ }^{17,18}$. Since then, these findings have been replicated in other species ${ }^{19,20}$ as well as in humans ${ }^{21}$.

In addition, during fetal development, glucocorticoids play a pivotal role in regulating the placenta that controls fetal exposure to the maternal environment ${ }^{22}$. The placenta expresses 11b-hydroxysteroid dehydrogenase type- 2 (11b-HSD2), which protects the fetus from excessive maternal glucocorticoids by metabolizing corticosterone into inactive 11-dehydrocorticosterone ${ }^{23}$. However, a significant reduction of this enzyme and its placental gene expression after repeated stress exposure in pregnancy has been observed ${ }^{24}$, so that it is not able to completely shield the fetus from glucocorticoid overexposure caused by the maternal stress reaction.

Crudo et al. ${ }^{25,26}$ reported global methylation changes in placental tissue and organs of the fetus after maternal betamethasone treatment in guinea-pigs. Global methylation remained altered in adult tissues of the offspring. Furthermore, the maternal betamethasone treatment also modifies DNA methylation and histone H3 lysine 9 acetylation in the fetal hippocampus ${ }^{27}$ and leads to differential $G r$ binding to a large number of different gene promotors and methylation of specific GREs in the fetal hippocampal $M r$ gene $^{26}$.

We hypothesized that $N r 3 c 1$ plays an important role in mediating the effects of stress on DNA methylation patterns systemically during the development and that these alterations mediate phenotypic effects of altered stress and glucocorticoid levels. We also reasoned that these methylation changes occur across several tissues and are not exclusive to the brain. We focused on fetal placenta since it is one of the few noninvasive sources of early life biological material at birth beside blood ${ }^{28,29}$ and perhaps one of the few biological sources that enable correlating DNA methylation at birth and later phenotypes in the same living animal.

As placental tissue is usually consumed by the mouse dam soon after birth, it is impossible to collect it in a natural setting. There are few studies linking characteristics of placenta and behavior of offspring in adulthood and they usually assign different litters to sample tissues and the behavioral tests (e.g., ${ }^{30,31}$ ).

We succeeded in establishing an elaborate procedure of caesarian section $^{32}$ that allowed the pups to develop with minimal neurotoxic side effects and provided us with placental tissue from the same individuals. Due to an upbringing with foster dams, the animals developed into adulthood and were tested for behavioral phenotypes as adults. This allowed us to measure in the same animal the baseline DNA methylation pattern in placenta and behavioral phenotypes later in life. To our knowledge, the present study is the first one linking epigenetic features of fetal placental tissue directly to the behavior of the same animals in adulthood. Our data suggest broad and physiologically pertinent changes in DNA methylation in placenta that correlate with anxiety-like behavior in adulthood in response to $\mathrm{Nr} 3 \mathrm{cl}$ deficiency.

\section{Methods}

Full details of all methods can be found in the supplementary file.

All procedures complied with the regulations covering animal experimentation within the EU (European Communities Council Directive 86/609/EEC) as well as national and local authorities (Regierungspräsidium Karlsruhe, Germany).

\section{Animals and tissue collection}

Acclimatized female $\mathrm{Nr} 3 \mathrm{cl}^{+/+}$and male $\mathrm{Nr}_{3} \mathrm{cl}^{+/-}$mice were used for breeding. $\mathrm{Nr} 3 \mathrm{cl}^{+/-}$animals were originally generated as described by Tronche et al. ${ }^{14,33}$. Pregnant females delivered by caesarean sections on E18.5 post conception. The procedure was completed within $10-20 \mathrm{~min}$ (11.5 min in average) using xenon gas and isoflurane anesthesia in order to protect the pups' brain and heart from hypoxia-induced damage ${ }^{32}$. The fetal placental tissue was kept on dry ice and stored at $-80{ }^{\circ} \mathrm{C}$ immediately after dissection. After caesarean sections, the biological dams were sacrificed and experienced C57BL/ $6 \mathrm{~N}$ foster dams whose own litter was aged between PND (postnatal day) 1 and PND 4 were used to raise the pups. The transfer into the nest of the lactating foster dam took place within $15 \mathrm{~min}$ after birth. The original pups were mixed with litter and urine of the foster dam to pick up her scent and so improving acceptance by the foster dam. We pooled the samples of individual placentae for the 
genome-wide epigenetic analysis to 3 groups of placental tissue per sex/genotype combination (Table S1).

Adult frontal cortex was collected from a different cohort of male GR-i animals (according to ref. ${ }^{34}$ ). These animals expressed GR antisense mRNA and consequently exhibited a decrease in GR-specific binding as well as GR mRNA levels in the frontal cortex ${ }^{35}$. Eight samples of frontal cortex were pooled and analyzed via ChIPbisulfite-Seq (for a more detailed description please see supplementary methods).

\section{Behavior}

Behavioral tests (Novel Cage, Open Field, Forced Swim Test, Hot Plate Test, Learned Helplessness and Dark Light Box as described elsewhere ${ }^{10,36}$ ) were performed by a completely blinded investigator. Maternal care was monitored from PND2 to PND8 for $24 \mathrm{~h}$ a day and rated via instantaneous sampling with a detailed ethogram adapted by Coutellier et al. ${ }^{37}$. Maternal care did not significantly differ between the dams (see Table S2 and Table S3).

\section{Statistics}

All analyses of variance (ANOVA) were conducted using the general linear model (GLM). Whenever the assumptions of parametric analysis were not met by graphical examination of homoscedasticity, raw data were transformed according to the Box-Cox-method or an outlier analysis was performed. All analyses of variance were based on a $2 \times 2$ factorial design with factors "sex" and "genotype". Two-way univariate ANOVAs were performed for weight of adrenals, spleens, birth weight, length of the newborn, rearing of novel cage, hot plate, sub-tests of dark-light-box, FST, learned helplessness, and methylation validation with pyrosequencing. For open field test and body weights over time, a two-way repeated measurements ANOVA was conducted with the factor "time". Linear Pearson product-moment coefficient was applied to determine correlations between DNA methylation and behavioral measures. Spearman rho coefficient was used for correlating number of failures and escape latency in the learned helplessness paradigm. Moderation effects were tested using Hayes Process macro for SPSS $^{38}$. A MANOVA with subsequent univariate two-way ANOVAs and $t$-tests were performed for the analysis of global methylation levels. For DNA methylation analysis and annotation, the $\mathrm{R}$ package "methylkit" was used ${ }^{39}$, correcting for multiple testing using Benjamini-Hochberg false discovery rate (FDR). A non-parametric $\chi^{2}$-test was calculated to detect differences between all 19 foster dams in maternal care behavior. The hypergeometric test as well as the binomial test was applied to test significant overlaps. Adjusted $p$ value $\leq 0.05$ was set as threshold for statistical significance except for genome wide DNA methylation data which was set at 0.2. Statistical analyses were performed using SPSS 21.0 software package for Windows.

\section{Capture bisulfite sequencing and DNA methylation mapping}

SeqCap Epi Enrichment System (Roche-NimbleGen) performed at the Institute de recherches cliniques de Montréal was used for targeted bisulfite sequencing of promoters and enhancers.

\section{Analysis of differentially methylated cytosines}

FastQC assessed sequencing scores and other quality metrics. Methylation levels and coverage levels were extracted with methratio.py command in Bsmap. Differential methylation cytosines were analyzed with methylKit $\mathrm{R}$ package ${ }^{39}$ with FDR threshold of 0.2 . Differentially methylated positions were annotated with $\mathrm{HOMER}^{40}$.

\section{Results \\ Effects of $\mathrm{Nr} 3 \mathrm{C}^{+/-}$deficiency on behavior \\ $\mathrm{Nr}_{3} \mathrm{C}^{+/-}$mice display an increase in anxiety-like behavior as measured by the dark-light box}

$N r 3 c 1$ depletion significantly increased anxiety-like behavior in all parameters monitored in the dark-light box in both sexes (Fig. 1a-d). Nr3c1 animals spent less time in the light compartment which is considered a measure of anxiety-like behavior in rodents. Maternal care, novel cage test, locomotor activity, depressive-like behaviors such as forced swim test and learned helplessness, body weight, adrenal and spleen size were not affected by $N r 3 c 1$ deficiency (please see supplementary results).

\section{Nr3c1 deficiency affects DNA methylation in a sex-dependent manner}

The genome wide state of methylation of promoters and enhancers was mapped using capture bisulfite sequencing (Fig. 2) and validated by pyro-sequencing (Fig. S1). In contrast to wild type animals which exhibit a small number of different methylation sites between sexes ${ }^{6}$ (see Fig. 2a, d), 2433 differentially methylated CpG sites were noted between males and females in Nr3c1 depleted animals (Table S4).

There was a multivariate interaction effect of sex*genotype on global methylation of cytosines at $\mathrm{CpG}$, $\mathrm{CHH}$, and CHG contexts $(p=0.013)$. Sex was significant for $\mathrm{CHH}\left(p=0.045, F_{3,8}=5.647\right)$ and almost reached significance for CpG $\left(p=0.072, F_{3,8}=4.276\right)$. Stratified for genotype, there was a statistical tendency for sex only in $N r 3 c 1^{+/-}$animals $\left(p=0.079, t_{4}=2.341\right)$ with males showing higher global methylation levels (see Fig. 2b).

Hypermethylated sites were more abundant in male $N r 3 c 1$ deficient animals (1023 in males vs 734 in females 


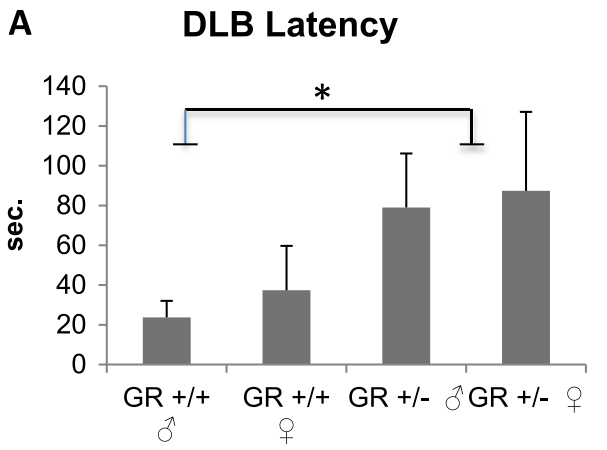

C

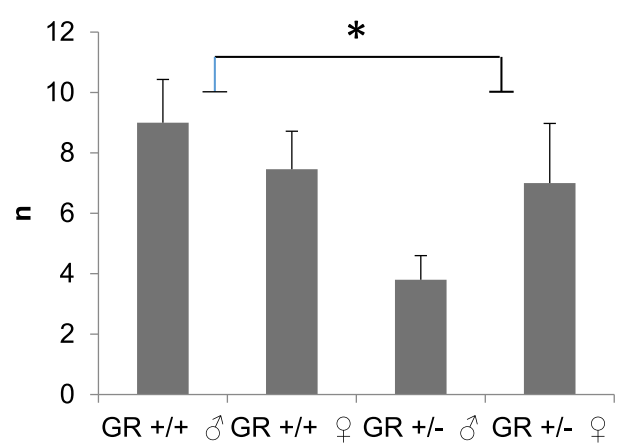

B

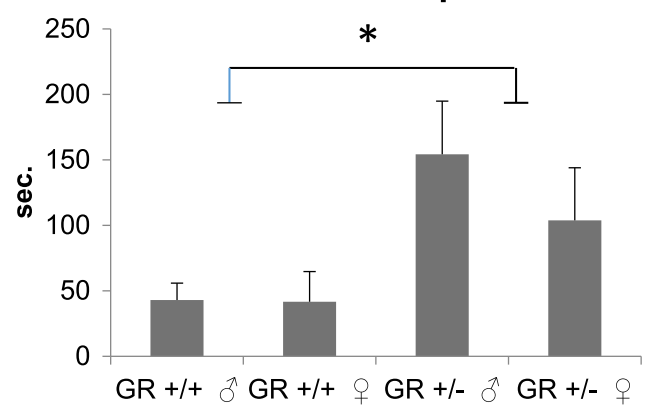

D

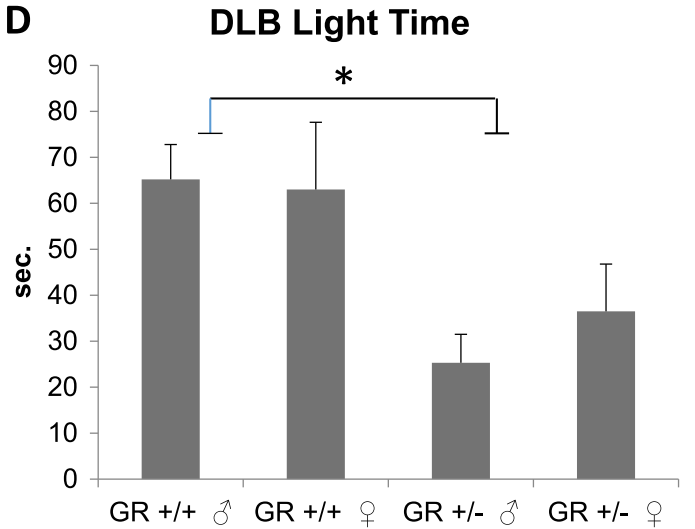

Fig. 1 Anxiety-like behavior measured by the dark-light-box. a Increased latency to enter the light department in $\mathrm{Gr}(\mathrm{Nr} 3 \mathrm{C} 1)^{+/-}$animals ( $p=$ $\left.0.011, F_{3,44}=7.047\right)$. $\mathbf{b}$ Increased latency to explore the end of the compartment in $\mathrm{Gr}(\mathrm{Nr} 3 \mathrm{Cl})^{+/-}$animals $\left(p=0.008, F_{3,44}=7.798\right)$. $\mathbf{c}$ Significant decrease in the number of exits in $\operatorname{Gr}(\mathrm{Nr} 3 \mathrm{Cl})^{+/-}$animals $\left(p=0.013, F_{3,44}=6.646\right)$. $\mathbf{d}$ Decreased duration of time spent in the light area in $\mathrm{Gr}(\mathrm{Nr} 3 \mathrm{Cl})^{+\prime}$ animals ( $\left.p=0.001, F_{3,44}=14.059\right)$. Means \pm SEM

$(p=2.869 \mathrm{E}-12$, binomial test), see Fig. 2c, d and Fig. S2) while hypomethylated sites were more frequent in females (901 in females vs 664 in males $(p=1.139 \mathrm{E}-09$, binomial test)). There was however a significant overlap between sites that are hypermethylated (hypergeometric test $p=$ $4.983 \mathrm{E}-11$ ) or hypomethylated (hypergeometric test $p=$ $2.435 \mathrm{E}-06)$ in both males and females. Each one of the four groups clusters separately (see Fig. 2e).

$N r 3 c 1^{+l-}$ deficiency causes significant loss of methylation in 51 genes and gain of methylation in 73 genes in both sexes (annotation: 15 reads, delta beta $=0.2$, FDR $<$ $0.2)$; 95 genes were hypermethylated in males and hypomethylated in females while 53 genes were hypomethylated in males and hypermethylated in females (Table S5-8).

Significant sex-dependent methylation in the opposite direction at the same CG site (hypermethylation in males and hypomethylation in females and vice versa) was detected in 10 sites: Gm16853, Mir6998, Rgs22, Coro2b, Babam1, Hoxd9, Gmppa, Slc14a2, Lhcgr, Cacnb1. Seven CGs were differentially methylated in $N r 3 c 1^{+/-}$in the same direction in males and females: Paqr4,
4930487D11Rik, Rusc1, Fshr, 5033406009Rik, Sumo2, Mir7078 (see Table S9).

\section{Effects of $\mathrm{Nr} 3 \mathrm{c} 1$ heterozygosity and sex on methylation state of candidate genes}

We then examined methylation in a shortlist of genes that were previously associated with early life stress and psychiatric disorders as well as placental functioning: Ank3, Avp, Avpr1a, Avpr1b, Bdnf, Cacna1c, Cyp11b1, Cyp11b2, Fkbp5, Hsd11b1, Igf2, Morc1, Nr3c1, Oxt, Oxtr, Pclo, Slc6a4.

There is no main or interaction effect of $N r 3 c 1$ genotype on methylation of $N r 3 c 1$. For Igf2, there is a significant main effect of sex on methylation $\left(p<0.001, F_{3,8}=\right.$ 25.378 ) with lower methylation in females. A significant sex*genotype-interaction was found for Hsd11b1 $\left(F_{3,8}=\right.$ 32.883, $p<0.001)$ and Cacna1c $\left(F_{3,8}=5,689 ; p=0.044\right)$ but no main effect of either sex or genotype. However, $N r 3 c 1$ deficiency significantly affected methylation of the proximal GC regulator $F k b p 5$ which is significantly hypomethylated in both male and female $\mathrm{Nr}_{3} \mathrm{c1}^{+/-}$mice $\left(F_{3,8}=10.537, p=0.012\right)$ (see Table S10). 


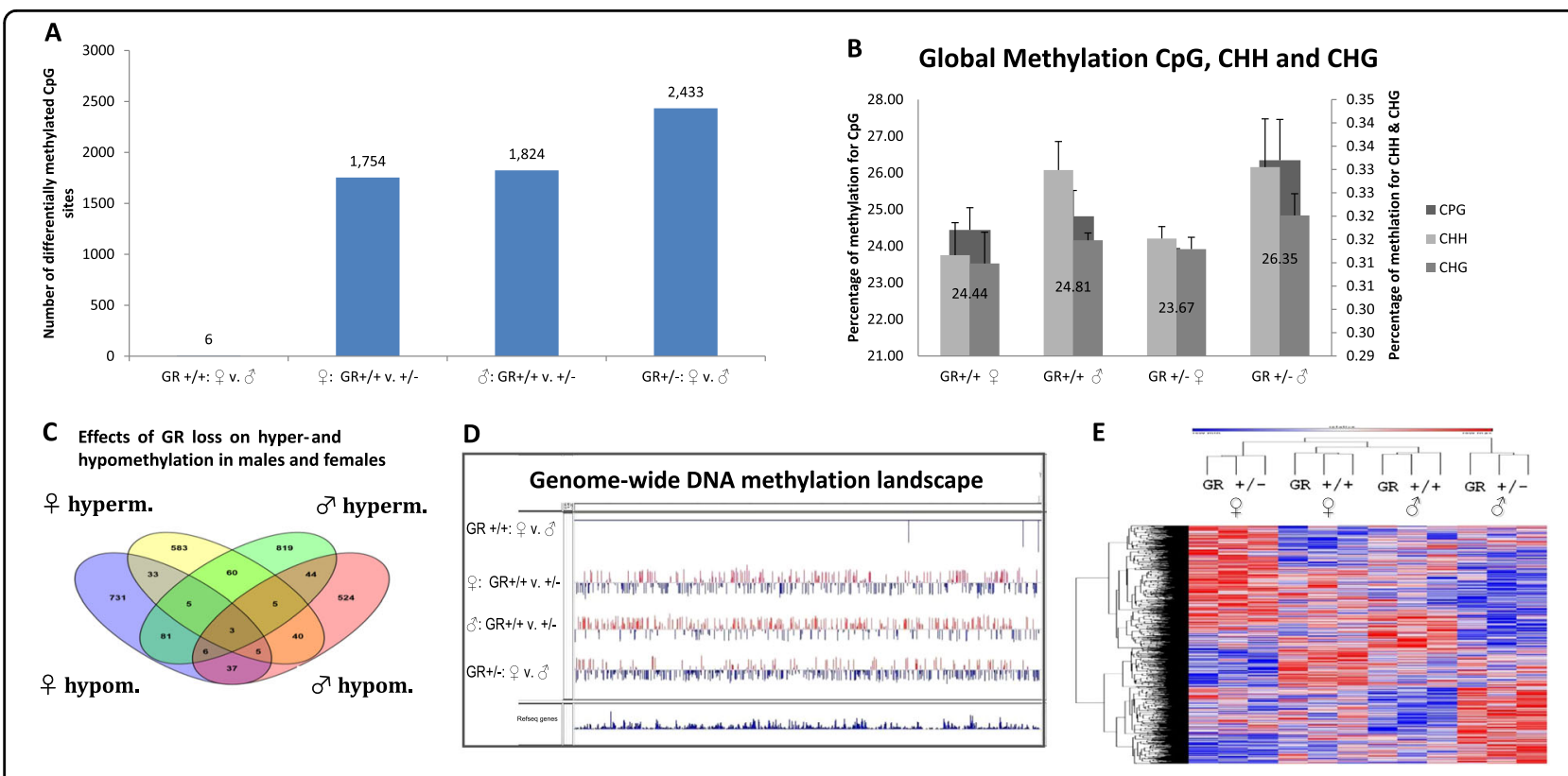

Fig. 2 Sex-dependent effects of $\mathrm{Nr} 3 \mathrm{c} \mathbf{1}$ deficiency. a Number of differentially methylated $\mathrm{CpG}$ sites for the comparisons of female $\mathrm{vs}$ male $\mathrm{Nr} 3 \mathrm{C} \mathrm{1}^{+1}$

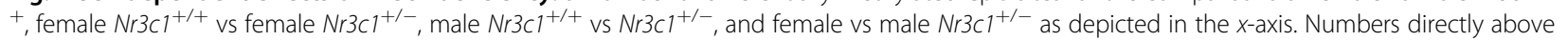
the graph bars represent absolute numbers of differentially methylated $\mathrm{CpG}$ sites. b Global methylation of $\mathrm{CpG}, \mathrm{CHH}$, and CHG sites in wildtype and $\mathrm{Nr3cl}$ knockouts in both sexes. Numbers depicted directly on the graphs represent the percentage of CpG sites (see also $y$-axis to the left). The percentage of $\mathrm{CHH}$ and $\mathrm{CHG}$ sites are illustrated on the right $y$-axis. c Effects of $\mathrm{Nr} 3 \mathrm{Cl}$ loss on hyper- and hypomethylation in males and females. $\mathbf{d}$ Genome-wide methylation tracks for CpG sites using Integrative Genomics Viewer (Broad Institute). Comparison is shown for female vs male wildtype, female wild type vs female $\mathrm{Nr} 3 \mathrm{Cl}$ heterozygous, male wild type vs male $\mathrm{Nr} 3 \mathrm{Cl}$ heterozygous, and male vs female $\mathrm{Nr} 3 \mathrm{Cl}$ heterozygous animals. e DNA methylation landscape of $\mathrm{Nr} 3 \mathrm{Cl}$ deficiency in male and female placental DNA. Heatmap (row distance metric: Pearson correlation, average linkage) depicting the clustering of $6017 \mathrm{CpGs}$ that were differentially methylated $(q<0.2)$ between placentae of $\mathrm{Gr}^{+/+}$and $\mathrm{Gr}^{+/-}$fetuses of both sexes. Rows correspond to CpGs and columns to animals' genotype and sex. Here, group number 1-3 indicates three pooled samples of $\mathrm{Fr}^{+/+}$ males, number 4-6 stands for three pooled samples of $\mathrm{Gr}^{+/-}$males, 19-21 for three pooled samples of $\mathrm{Gr}^{+/+}$females, and 22-24 for three pooled samples of $\mathrm{Gr}^{+/-}$females. Red indicates higher methylation in a row and blue indicates lower methylation. See also Table S4-S9

\section{Functional analysis of sex-dependent alteration of methylation induced by $\mathrm{Nr} 3 \mathrm{c} 1$ deficiency}

We analyzed potential canonical pathways using ingenuity pathway analysis: a difference between males and females in the level of enrichment of DNA methylation alterations in $\mathrm{Nr} 3 \mathrm{cl}$ deficient mice was shown in central nervous system-related functions like Reelin and CREB signaling in neurons, whereas ubiquitous cell signaling pathways like the $\mathrm{Wnt} / \mathrm{Ca}+$ pathway coupled receptor were found to be similarly affected in both sexes (Fig. 3).

Beside cell signaling and developmental functions many molecules regulating neuronal and metabolic processes were associated with differentially methylated genes in both sexes (see Table S11) and the top upstream regulator was the central nervous system-specific gene Cannabinoid receptor 1 (CNR1) (see Table S12).

Five pathways directly related to central neurotransmission were enriched in genes that were hypermethylated in males and hypomethylated in females, whereas the pathways enriched for genes that were hypomethylated in males and hypermethylated in females included mainly hormonal and general cell signaling pathways. There are only two common canonical pathways for the 95 genes hypermethylated in males and at the same time hypomethylated in females and the 53 genes that were hypomethylated in males and hypermethylated in females: G-Protein Coupled Receptor Signaling and Goi signaling (see Fig. S3 and S4).

\section{Overlap in differentially methylated promoters in Nr3c1- deficient mice in fetal placenta and adult frontal cortex}

We examined whether there is an overlap between the genes differentially methylated in placenta of $N r 3 \mathrm{cl}^{+/-}$ male fetuses (based on capture sequencing analysis of the present study) and frontal cortex of adult male Gr-I mice from a separate study (unpublished, based on a ChIPbisulfite-Seq-analysis of GR-bound DNA methylation). 242 genes were differentially hyper- and hypomethylated in both tissues $(17.5 \%$ of the genes that are differentially methylated in male fetal $N r 3 c 1^{+/-}$placentae) $(p=$ 0.000388 , hypergeometric test, FDR $<0.2$; see Table S13).

Among others, specific neurotransmitter systems like serotonin and glutamate receptor signaling as well as neuronal developmental (e.g., netrin signaling) and neuroprotective (TNFR2-signaling) pathways were found enriched (see Fig. 4). 


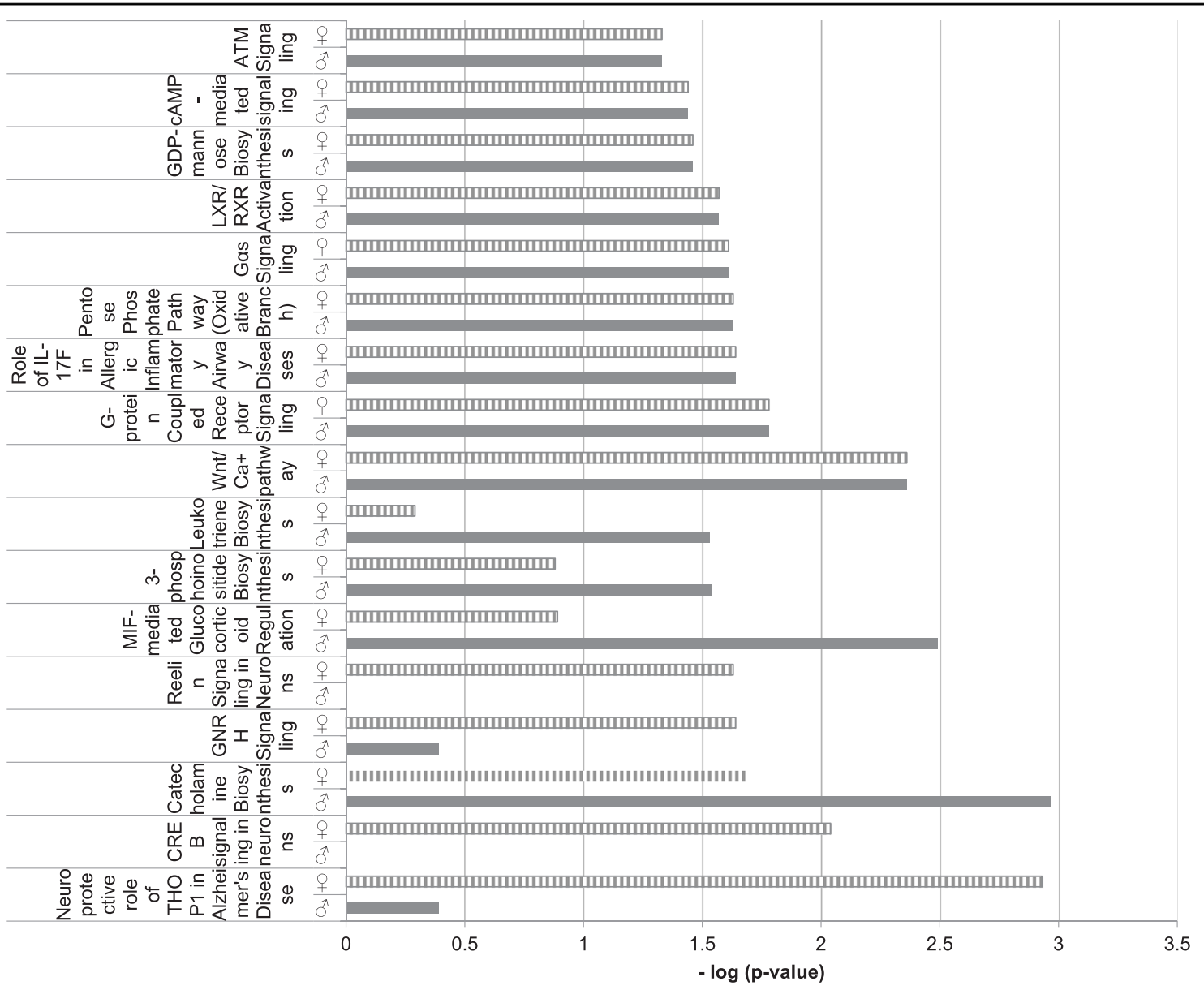

Fig. 3 Ingenuity pathway analysis. Selected canonical pathways for the comparison of methylation changes in males and females after $\mathrm{Nr} 3 \mathrm{Cl}$ loss. See also Table S11 and S12

Brain functions as well as metabolic-related functions were enriched in both tissues. Additionally, molecules known to be involved in fundamental central nervous functions (see Table S14), molecules involved in basal metabolic processes and oxidative stress as well as a considerable number of microRNA loci (see Table S15) were enriched with differentially methylated genes in placenta as well as frontal cortex. For both tissues, the transcription factors Fos and Atn1 were found as upstream regulators (see Table S16).

\section{Correlation between differentially methylated genes and anxiety-like behavior in $\mathrm{Nr} 3 \mathrm{Cl}$ deficient mice}

We correlated the quantitative levels of methylation of CG sites of genes commonly differentially methylated in both sexes (see Table S17) with quantitative behavioral scores for each individual mouse. The behavioral score of anxiety-like behavior of the adult males-but not females -yielded 5 significant correlations with Slc1a7, 9530052E02Rik, Itgb7, Tiam2, and Tspo (see Fig. 5a-f, adjusted $p=0.048$ ).
The correlation of methylation levels with depression as measured by the Forced Swim Test and Learned Helpless Paradigm as well as locomotor activity (Novel Cage and Open Field Test) did not lead to significant results consistent with the lack of impact of Nr3c1 heterozygosity on the latter behaviors (please see supplementary results).

\section{Moderation of levels of anxiety-like behavior in adulthood by differential DNA methylation in fetal placenta}

Finally, we assessed the moderating influence of candidate genes whose level of methylation is associated with the $N r 3 c 1$ genotype on anxiety-like behavior: $A n k 3, A v p$, Avpr1a, Avpr1b, Bdnf, Cacna1c, Cyp11b1, Cyp11b2, Fkbp5, Hsd11b1, Igf2, Morc1, Nr3c1, Oxt, Oxtr, Pclo, Slc6a4. Methylation of Fkbp5 significantly moderated $\mathrm{Nr} 3 \mathrm{c} 1$ influence on anxiety-like behavior in adulthood $\left(F_{1,8}=18.1987, p=0.0027\right)$. The higher Fkbp5's methylation level, the longer the animals spent in the light indicating a lower level of anxiety-like behavior. The average methylation of $F k b p 5$ was significantly reduced in both male and female $N r 3 c 1$ depleted animals (delta for 

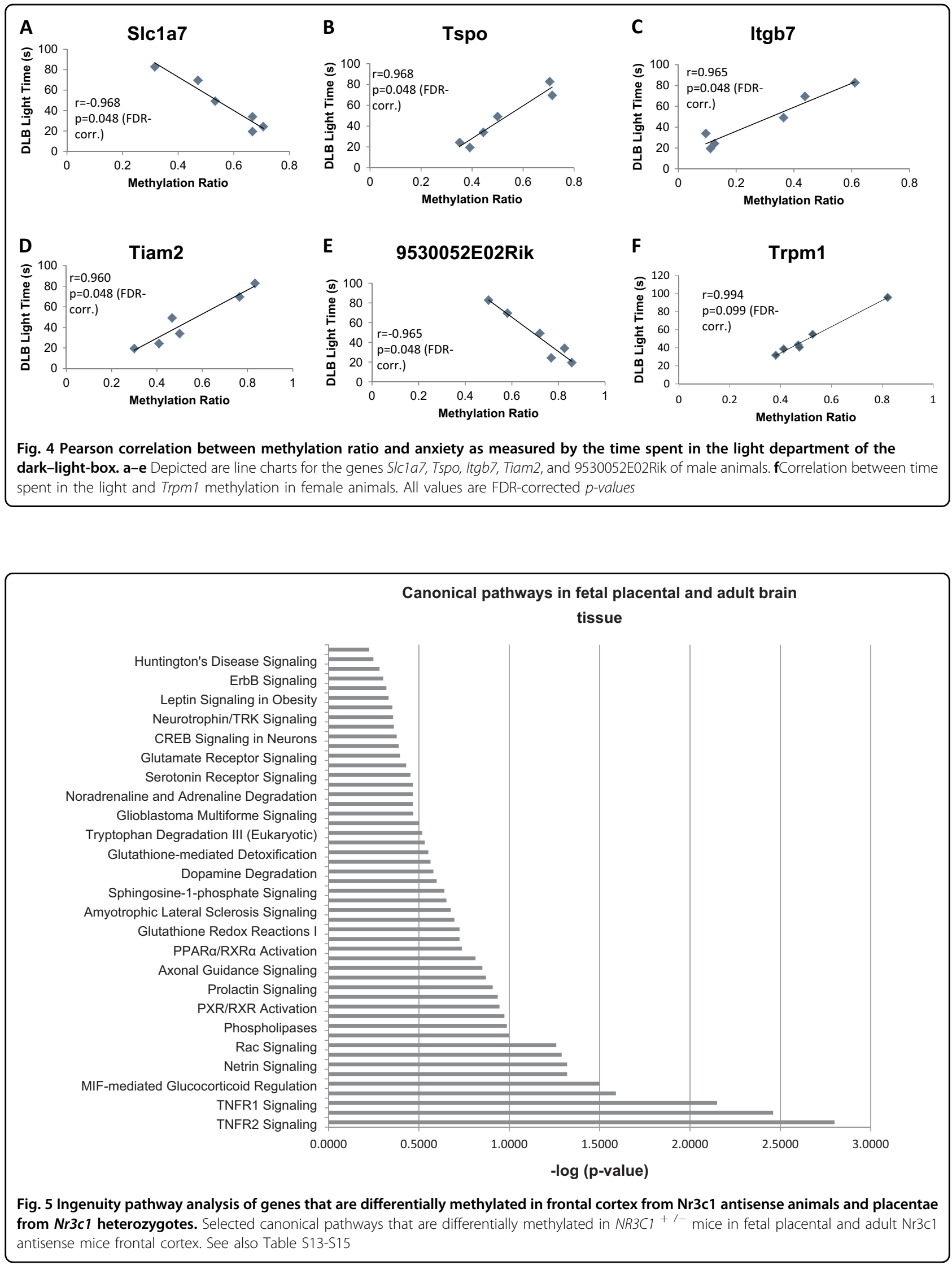
males $=-0.254$, females $=-0.341, \quad F_{3,8}=10.537, \quad p=$ 0.012 , see Table S10).

\section{Discussion}

Our study tests for the first time the hypothesis that $N r 3 c 1$ plays a causal role in shaping the DNA methylation profile of the fetus and that these changes in methylation triggered by $\mathrm{Nr} 3 \mathrm{cl} 1$ deficiency at birth are associated with phenotypic alterations. $N r 3 \mathrm{cl}^{+/-}$animals of both sexes showed a significant increase exclusively in anxiety-like behavior which is consistent with data derived from several different transgenic mouse models ${ }^{41,42}$. Two of the five genes whose methylation state in placenta significantly correlate with levels of anxiety-like behavior only in adult males but not females: Tspo and Slc1a7 are involved in central nervous system functions. Tspo polymorphism is linked to separation anxiety in depressive patients $^{43}$ and Slc1a7 inhibits glutamate receptor activity $^{44}$.

$N r 3 c 1$ deficiency dramatically enhances differences in DNA methylation between the sexes. This provides a possible mechanism for sex-dependent effects of prenatal stress that were previously reported (e.g., ${ }^{45-48}$ ). Although we examined DNA methylation in the placenta, which reflects prenatal experience, it should be noted that early postnatal stress also leads to sex-specific effects later in life and the role of $N r 3 c 1$ in these effects needs further study (e.g., ${ }^{49-54}$ ). In addition, it should be noted that perinatal gonadal hormones are capable of inducing changes in methylation patterns between the sexes in wild type animals as well ${ }^{55,56}$.

Nugent et al. ${ }^{57}$ examined the highly sexually dimorphic preoptic area of the rat hypothalamus. They report reduced activity of DNA methyltransferase in this as well as decreased DNA methylation and release of masculinizing genes from epigenetic repression. Gonadal steroids are physiologically released in the first days after birth. However, the sex differences in the fetal part of placenta of the present study were detected on day 18.5 postconception well before the influence of the postnatal testosterone and its metabolite estradiol. Furthermore, Nugent et al. ${ }^{57}$ describe an astonishingly small number of differentially methylated genes between the sexes exactly as reported in our study: there were only 6 sites differentially methylated between males and females of our wildtype control group vs 2433 sites between our Nr3c1heterozygous female and male animals. The fact that the differences in methylation between the sexes were dramatically enhanced in the heterozygous animals indicates a gene by sex effect on DNA methylation.

Furthermore, Nugent et al. ${ }^{57}$ found higher levels of global CpG-methylation in females in the preoptic area of the hypothalamus. This finding is different from our observation of elevated CpG-methylation in the male placenta of $\mathrm{Nr}_{3} \mathrm{cl}^{+/-}$animals. Since methylation is highly tissue-specific, this might explain the observed differences in global methylation between the two studies. However, it is possible that $N r 3 c 1$ deficiency changed the DNA methylation landscape independently of postnatal hormonal influence and switched the differences in global methylation between the sexes.

In addition, we examined the effect of sex and $N r 3 c 1$ deficiency on selected candidate genes that are known to be involved in responses to prenatal stress. The growth promoting hormone Igf2 was significantly less methylated in wild type females as compared to males. Mina et al. ${ }^{58}$ previously reported elevated Igf2 mRNA in fetal female as compared to male placenta of distressed mothers. Although we observed a sex effect on Igf2 DNA methylation, there was no effect of $N r 3 c 1$ deficiency. However, $H s d 11 b 1$, encoding a protein which converts cortisol to the inactive metabolite cortisone is hypermethylated in $\mathrm{Nr}_{3} \mathrm{cl}^{+/-}$males as compared to $\mathrm{Nr} 3 \mathrm{cl}^{+/-}$females and to wildtype males. Although Green et al. ${ }^{59}$ did not find a sex difference in $H s d 11 b 1$ methylation in human placenta, low methylation of $H s d 11 b 1$ was associated with the risk of being born large for gestational age. Similarly, a sex*genotype interaction effect on DNA methylation was found for Slc6a4 and Cacna1 which were previously associated with stress-related disorders ${ }^{60}$.

$F k b p 5$, the proximal regulator of the glucocorticoid receptor, was hypomethylated in placental tissue of our $\mathrm{Nr}_{3} \mathrm{Cl}^{+/-}$animals. This result is in line with findings of St-Cyr et al. ${ }^{61}$ who report hypomethylation of $F k p b 5$ in the amygdala of adult female offspring as well as increased ACTH stress reactivity after exposure to the prenatal odor of a predator. $F k b p 5$ polymorphisms enhance the risk of developing stress-related disorders in adulthood after early traumatic experiences ${ }^{62}$. This effect is allele-specific and depends on epigenetic changes in the glucocorticoid response elements of $F k b p 5^{63}$. Most interestingly, we also found a significant moderation effect of Fkbp5's CGmethylation level on anxiety-like behavior in adulthood. Hartmann et al. ${ }^{64}$ succeeded in reducing an anxious-like phenotype caused by an overexpression of FKBP51 in the basolateral amygdala by applying a highly selective FKBP51 point mutation antagonist. A new specific antagonist of FKBP5-SAFIT2-reduced anxiety-like behavior even when administered peripherally. It would be intriguing to test if SAFIT2 could also rescue the anxious-like phenotype of our $N r 3 \mathrm{Cl}^{+/-}$animals.

Besides hormonal receptors and microRNAs, several genes that are differentially methylated in both sexes in the same CGs when $N r 3 c 1$ is deficient are also associated with neuronal and mental disorders (6 out of 17 CGs). Four of these genes were differentially methylated in the opposite direction: Babam1 has been linked to schizophrenia $^{65}$, Cacnb1 to autism ${ }^{66}$ and depression ${ }^{67}$, Coro $2 b$, a 
candidate gene for ciliopathies to intellectual disability ${ }^{68}$ and Gmppa to intellectual disability and autonomic dysfunction ${ }^{69}$. Two genes that are differentially methylated in the same direction in males and females are Rusc1 whose gene product serves as neuronal adaptor protein ${ }^{70}$ and Paqr4 which was recently associated with the development of epilepsy ${ }^{71}$.

The functional pathway analysis revealed that the top upstream regulator of differentially methylated genes in both sexes in response to $N r 3 c 1$ deficiency is the Cannabinoid receptor 1 (CNR1) gene which is expressed in the central nervous system and is known to be associated with exploratory drive, anxiety and stress response ${ }^{72}$. Most interestingly, the endocannabinoid receptor is also discussed as a possible target for anxiolytic drugs ${ }^{73}$.

To our knowledge, there is only one study that examined the correlation of gene expression in response to prenatal stress in placenta with adult behavior: Mueller and $\mathrm{Bale}^{31}$ found a maladaptive stress responsivity in adulthood in males exposed to prenatal stress, which was linked to a significantly increased gene expression of PPAR $\alpha$, IGFBP-1, HIF3 $\alpha$, and GLUT4 in male placenta. The authors propose a mechanism by which the expression of PPAR $\alpha$ is increased by glucocorticoids ${ }^{74}$, which in turn induces expression of IGFBP- $1^{75}$. Interestingly, PPARY and IGFBP-2 are differentially methylated in frontal cortex as well as placenta of $N r 3 c 1$ deficient males of our study.

PPAR $y$-agonists that are usually used for treating type- 2 diabetes have been reported to exhibit antidepressant effects as well ${ }^{76}$. IGFBP-2 has also been linked to diabetes and depression in peripheral ${ }^{77}$ and central nervous tissue $^{78}$. This illustrates the potential overlap between psychiatric disorders and metabolic dysfunctions as reported before $^{79}$.

Our data point to the potential utility of placental DNA methylation markers for early diagnosis of prenatal stress and for prediction of the emergence of behavioral disorders later in life. Thus, further evaluation of placental tissue in mice as well as humans, which is easily available after delivery, as a possible source for the predictor of risk for adult psychiatric disorders at birth seems very promising.

\section{Acknowledgements \\ This work was supported by an Era-Net Neuron grant to M.S., P.G., and M.D. and MOP-42411 from the Canadian Institute of Health Research to M.S. E.L. was supported by The Richard and Edith Strauss Postdoctoral Fellowship in Medicine, McGill University. The authors thank Carsten Pilger, PhD, for his excellent technical advice. The authors are grateful to Madeleine Saitta for outstanding animal caretaking.}

\section{Author details}

${ }^{1}$ Central Institute of Mental Health Mannheim (ZI), Medical Faculty of Mannheim, University of Heidelberg, J5, 68159 Mannheim, Germany. ${ }^{2}$ Department of Pharmacology \& Therapeutics, McGill University, Montreal, QC H3G 1Y6, Canada. ${ }^{3}$ Sackler Program for Epigenetics and Psychobiology, McGill
University, Montreal, QC H3G 1Y6, Canada. ${ }^{4}$ Department of Pharmacological and Biomolecular Sciences, University of Milan, Via Balzaretti, 9, I-20133 Milan, Italy. ${ }^{5}$ Inserm, U894, Centre de Psychiatrie et Neurosciences, 75014 Paris,

France. ${ }^{6}$ Université Paris Descartes, UMRS894, 75014 Paris, France

Conflict of interest

The authors declare that they have no conflict of interest.

\section{Publisher's note}

Springer Nature remains neutral with regard to jurisdictional claims in published maps and institutional affiliations.

Supplementary Information accompanies this paper at (https://doi.org/ 10.1038/s41398-018-0348-7). Raw data are archived in the Gene Expression Omnibus under the following accession numbers: GSE123188 and GSE123460.

Received: 6 October 2018 Accepted: 13 November 2018

Published online: 17 January 2019

\section{References}

1. Holsboer, F. The corticosteroid receptor hypothesis of depression. Neuropsychopharmacology 23, 477-501 (2000).

2. Brunton, P. J. \& Russell, J. A. Prenatal social stress in the rat programmes neuroendocrine and behavioural responses to stress in the adult offspring: sex-specific effects. J. Neuroendocrinol. 22, 258-271 (2010).

3. Koehl, M., Barbazanges, A., Le Moal, M. \& Maccari, S. Prenatal stress induces a phase advance of circadian corticosterone rhythm in adult rats which is prevented by postnatal stress. Brain Res. 759, 317-320 (1997).

4. Laloux, C. et al. Anxiety-like behaviour and associated neurochemical and endocrinological alterations in male pups exposed to prenatal stress. Psychoneuroendocrinology 37, 1646-1658 (2012).

5. Morley-Fletcher, S., Rea, M., Maccari, S. \& Laviola, G. Environmental enrichment during adolescence reverses the effects of prenatal stress on play behaviour and HPA axis reactivity in rats. Eur. J. Neurosci. 18, 3367-3374 (2003).

6. Hiroi, R., Carbone, D. L., Zuloaga, D. G., Bimonte-Nelson, H. A. \& Handa, R. J. Sexdependent programming effects of prenatal glucocorticoid treatment on the developing serotonin system and stress-related behaviors in adulthood. Neuroscience 320, 43-56 (2016).

7. Talge, N. M., Neal, C. \& Glover, V. Antenatal maternal stress and long-term effects on child neurodevelopment: how and why? J. Child Psychol. Psychiatry 48, 245-261 (2007).

8. St-Cyr, S. \& McGowan, P. O. Adaptation or pathology? The role of prenatal stressor type and intensity in the developmental programing of adult phenotype. Neurotoxicol. Teratol. 66, 113-124 (2018).

9. Bock, J., Wainstock, T., Braun, K. \& Segal, M. Stress in utero: prenatal programming of brain plasticity and cognition. Biol. Psychiatry 78, 315-326 (2015).

10. Vogt, M. A. et al. Inducible forebrain-specific ablation of the transcription factor Creb during adulthood induces anxiety but no spatial/contextual learning deficits. Front. Behav. Neurosci. 8, 407 (2014).

11. Barbazanges, A., Piazza, P. V., Le Moal, M. \& Maccari, S. Maternal glucocorticoid secretion mediates long-term effects of prenatal stress. J. Neurosci. 16, 3943-3949 (1996).

12. Darnaudery, M. \& Maccari, S. Epigenetic programming of the stress response in male and female rats by prenatal restraint stress. Brain Res. Rev. 57, 571-585 (2008).

13. Boersma, G. J. et al. Prenatal stress decreases Bdnf expression and increases methylation of Bdnf exon IV in rats. Epigenetics 9, 437-447 (2014).

14. Tronche, F. et al. Disruption of the glucocorticoid receptor gene in the nervous system results in reduced anxiety. Nat. Genet. 23, 99-103 (1999).

15. Ridder, S. et al. Mice with genetically altered glucocorticoid receptor expression show altered sensitivity for stress-induced depressive reactions. J. Neurosci. 25, 6243-6250 (2005).

16. Finotto, S. et al. Analysis of mice carrying targeted mutations of the glucocorticoid receptor gene argues against an essential role of glucocorticoid signalling for generating adrenal chromaffin cells. Development 126, 2935-2944 (1999) 
17. Weaver, I. C. et al. Epigenetic programming by maternal behavior. Nat. Neurosci. 7, 847-854 (2004).

18. Weaver, I. C. et al. Reversal of maternal programming of stress responses in adult offspring through methyl supplementation: altering epigenetic marking later in life. J. Neurosci. 25, 11045-11054 (2005).

19. Rubenstein, D. R. et al. Sex-specific fitness effects of unpredictable early life conditions are associated with DNA methylation in the avian glucocorticoid receptor. Mol. Ecol. 25, 1714-1728 (2016).

20. Liberman, S. A., Mashoodh, R., Thompson, R. C., Dolinoy, D. C. \& Champagne, F. A. Concordance in hippocampal and fecal $\mathrm{Nr} 3 \mathrm{C} 1$ methylation is moderated by maternal behavior in the mouse. Ecol. Evol. 2, 3123-3131 (2012).

21. McGowan, P. O. et al. Epigenetic regulation of the glucocorticoid receptor in human brain associates with childhood abuse. Nat. Neurosci. 12, 342-348 (2009).

22. Jansson, T. \& Powell, T. L. Role of the placenta in fetal programming: underlying mechanisms and potential interventional approaches. Clin. Sci. 113, 1-13 (2007).

23. Benediktsson, R., Calder, A. A., Edwards, C. R. \& Seckl, J. R. Placental 11 betahydroxysteroid dehydrogenase: a key regulator of fetal glucocorticoid exposure. Clin. Endocrinol. 46, 161-166 (1997).

24. Welberg, L. A., Thrivikraman, K. V. \& Plotsky, P. M. Chronic maternal stress inhibits the capacity to up-regulate placental 11 beta-hydroxysteroid dehydrogenase type 2 activity. J. Endocrinol. 186, R7-R12 (2005).

25. Crudo, A. et al. Prenatal synthetic glucocorticoid treatment changes DNA methylation states in male organ systems: multigenerational effects. Endocrinology 153, 3269-3283 (2012).

26. Crudo, A. et al. Effects of antenatal synthetic glucocorticoid on glucocorticoid receptor binding, DNA methylation, and genome-wide mRNA levels in the fetal male hippocampus. Endocrinology 154, 4170-4181 (2013).

27. Crudo, A. et al. Glucocorticoid programming of the fetal male hippocampal epigenome. Endocrinology 154, 1168-1180 (2013).

28. Nieratschker, V. et al. MORC1 exhibits cross-species differential methylation in association with early life stress as well as genome-wide association with MDD. Transl. Psychiatry 4, e429 (2014).

29. Suarez, A. et al. The epigenetic clock at birth: associations with maternal antenatal depression and child psychiatric problems. J. Am. Acad. Child Adolesc. Psychiatry 57, 321-328 (2018).

30. Wyrwoll, C. et al. Fetal brain 11beta-hydroxysteroid dehydrogenase type 2 selectively determines programming of adult depressive-like behaviors and cognitive function, but not anxiety behaviors in male mice. Psychoneuroendocrinology 59, 59-70 (2015).

31. Mueller, B. R. \& Bale, T. L. Sex-specific programming of offspring emotionality after stress early in pregnancy. J. Neurosci. 28, 9055-9065 (2008).

32. Ruder, A. M., Schmidt, M., Ludiro, A., Riva, M. A. \& Gass, P. A technique for administering xenon gas anesthesia during surgical procedures in mice. Lab Anim. 43, 405-409 (2014).

33. Tronche, F., Kellendonk, C., Reichardt, H. M. \& Schutz, G. Genetic dissection of glucocorticoid receptor function in mice. Curr. Opin. Genet. Dev. 8, 532-538 (1998).

34. Pepin, M. C., Pothier, F. \& Barden, N. Impaired type II glucocorticoid-receptor function in mice bearing antisense RNA transgene. Nature 355, 725-728 (1992).

35. Froger, N. et al. Neurochemical and behavioral alterations in glucocorticoid receptor-impaired transgenic mice after chronic mild stress. J. Neurosci. 24, 2787-2796 (2004).

36. Weber, T. et al. Adult AMPA GLUA1 receptor subunit loss in 5-HT neurons results in a specific anxiety-phenotype with evidence for dysregulation of 5-HT neuronal activity. Neuropsychopharmacology 40, 1471-1484 (2015).

37. Coutellier, L., Friedrich, A. C., Failing, K., Marashi, V. \& Wurbel, H. Effects of rat odour and shelter on maternal behaviour in C57BL/6 dams and on fear and stress responses in their adult offspring. Physiol. Behav. 94, 393-404 (2008).

38. Hayes, A. F. Introduction to Mediation, Moderation and Conditional Process Analysis (Guilford Press, New York, 2013).

39. Akalin, A. et al. methylKit: a comprehensive $\mathrm{R}$ package for the analysis of genome-wide DNA methylation profiles. Genome Biol. 13, R87 (2012).

40. Xi, Y. \& Li, W. BSMAP: whole genome bisulfite sequence MAPping program. BMC Bioinformatics 10, 232 (2009).

41. Arnett, M. G., Kolber, B. J., Boyle, M. P. \& Muglia, L. J. Behavioral insights from mouse models of forebrain- and amygdala-specific glucocorticoid receptor genetic disruption. Mol. Cell. Endocrinol. 336, 2-5 (2011).
42. Salmaso, N. et al. Fibroblast growth factor 2 modulates hypothalamic pituitary axis activity and anxiety behavior through glucocorticoid receptors. Biol. Psychiatry 80, 479-489 (2016).

43. Costa, B. et al. Role of translocator protein $(18 \mathrm{kDa})$ in adult separation anxiety and attachment style in patients with depression. Curr. Mol. Med. 12, 483-487 (2012).

44. Zhou, Y. \& Danbolt, N. C. GABA and glutamate transporters in brain. Front. Endocrinol. 4, 165 (2013).

45. Barbie-Shoshani, Y., Shoham, S., Bejar, C. \& Weinstock, M. Sex-specific effects of prenatal stress on memory and markers of neuronal activity in juvenile rats. Dev. Neurosci. 38, 206-219 (2016).

46. Grundwald, N. J. \& Brunton, P. J. Prenatal stress programs neuroendocrine stress responses and affective behaviors in second generation rats in a sexdependent manner. Psychoneuroendocrinology 62, 204-216 (2015).

47. Luoni, A. et al. Sex-specific effects of prenatal stress on Bdnf expression in response to an acute challenge in rats: a role for Gadd45beta. Mol. Neurobiol. 53, 7037-7047 (2016).

48. Glover, V. \& Hill, J. Sex differences in the programming effects of prenatal stress on psychopathology and stress responses: an evolutionary perspective. Physiol. Behav. 106, 736-740 (2012).

49. Dow-Edwards, D., Frank, A., Wade, D., Weedon, J. \& Izenwasser, S. Sexuallydimorphic alterations in cannabinoid receptor density depend upon prenatal/ early postnatal history. Neurotoxicol. Teratol. 58, 31-39 (2016).

50. Lesse, A., Rether, K., Groger, N., Braun, K. \& Bock, J. Chronic postnatal stress induces depressive-like behavior in male mice and programs second-hit stress-induced gene expression patterns of OxtR and AvpR1a in adulthood. Mol. Neurobiol. 54, 4813-4819 (2017).

51. Massart, R. et al. Early life adversity alters normal sex-dependent developmental dynamics of DNA methylation. Dev. Psychopathol. 28, 1259-1272 (2016).

52. Loi, M. et al. Effects of early-life stress on cognitive function and hippocampal structure in female rodents. Neuroscience 342, 101-119 (2017).

53. Nishi, M., Horii-Hayashi, N. \& Sasagawa, T. Effects of early life adverse experiences on the brain: implications from maternal separation models in rodents. Front. Neurosci. 8, 166 (2014).

54. Oyola, M. G. \& Handa, R. J. Hypothalamic-pituitary-adrenal and hypothalamic-pituitary-gonadal axes: sex differences in regulation of stress responsivity. Stress 20, 476-494 (2017).

55. Aranda, G. et al. Effects of sex steroids on the pattern of methylation and expression of the promoter region of estrogen and androgen receptors in people with gender dysphoria under cross-sex hormone treatment. J. Steroid Biochem. Mol. Biol. 172, 20-28 (2017).

56. Mani, S. T. \& Thakur, M. K. In the cerebral cortex of female and male mice, amyloid precursor protein (APP) promoter methylation is higher in females and differentially regulated by sex steroids. Brain Res. 1067, 43-47 (2006).

57. Nugent, B. M. et al. Brain feminization requires active repression of masculinization via DNA methylation. Nat. Neurosci. 18, 690-697 (2015).

58. Mina, T. H., Raikkonen, K., Riley, S. C., Norman, J. E. \& Reynolds, R. M. Maternal distress associates with placental genes regulating fetal glucocorticoid exposure and IGF2: role of obesity and sex. Psychoneuroendocrinology 59, 112-122 (2015).

59. Green, B. B. et al. The role of placental 11-beta hydroxysteroid dehydrogenase type 1 and type 2 methylation on gene expression and infant birth weight. Biol. Reprod. 92, 149 (2015).

60. Cordova-Palomera, A. et al. Genome-wide methylation study on depression: differential methylation and variable methylation in monozygotic twins. Transl. Psychiatry 5, e557 (2015).

61. St-Cyr, S., Abuaish, S., Sivanathan, S. \& McGowan, P. O. Maternal programming of sex-specific responses to predator odor stress in adult rats. Horm. Behav. 94, $1-12$ (2017).

62. Yeo, S. et al. The influence of FKBP5 genotype on expression of FKBP5 and other glucocorticoid-regulated genes, dependent on trauma exposure. Genes Brain Behav. 16, 223-232 (2017).

63. Klengel, $T$. et al. Allele-specific FKBP5 DNA demethylation mediates genechildhood trauma interactions. Nat. Neurosci. 16, 33-41 (2013).

64. Hartmann, J. et al. Pharmacological inhibition of the psychiatric risk factor FKBP51 has anxiolytic properties. J. Neurosci. 35, 9007-9016 (2015).

65. Hass, J. et al. A genome-wide association study suggests novel loci associated with a schizophrenia-related brain-based phenotype. PLOS ONE 8, e64872 (2013). 
66. Kichukova, T. M., Popov, N. T., Ivanov, I. S. \& Vachev, T. I. Profiling of circulating serum MicroRNAs in children with autism spectrum disorder using stem-loop qRT-PCR assay. Folia Med. 59, 43-52 (2017).

67. Malki, K. et al. Antidepressant-dependent mRNA changes in mouse associated with hippocampal neurogenesis in a mouse model of depression. Pharmacogenet. Genomics 22, 765-776 (2012).

68. Castro-Sanchez, S. et al. Whole exome sequencing as a diagnostic tool for patients with ciliopathy-like phenotypes. PLOS ONE 12, e0183081 (2017).

69. Gold, W. A. et al. A novel mutation in GMPPA in siblings with apparent intellectual disability, epilepsy, dysmorphism, and autonomic dysfunction. Am. J. Med. Genet. A 173, 2246-2250 (2017).

70. MacDonald, J. I. et al. Nesca, a novel neuronal adapter protein, links the molecular motor kinesin with the pre-synaptic membrane protein, syntaxin-1, in hippocampal neurons. J. Neurochem. 121, 861-880 (2012).

71. Zhang, S., Kou, Y., Hu, C. \& Han, Y. MicroRNA profiling in the dentate gyrus in epileptic rats: the role of miR-187-3p. Medicine 96, e6744 (2017).

72. Qin, Z. et al. Chronic stress induces anxiety via an amygdalar intracellular cascade that impairs endocannabinoid signaling. Neuron 85, 1319-1331 (2015).

73. Patel, S., Hill, M. N., Cheer, J. F., Wotjak, C. T. \& Holmes, A. The endocannabinoid system as a target for novel anxiolytic drugs. Neurosci. Biobehav. Rev. 76, 56-66 (2017).
74. Lemberger, $\mathrm{T}$. et al. Regulation of the peroxisome proliferator-activated receptor alpha gene by glucocorticoids. J. Biol. Chem. 269, 24527-24530 (1994).

75. Degenhardt, T., Matilainen, M., Herzig, K. H., Dunlop, T. W. \& Carlberg, C. The insulin-like growth factor-binding protein 1 gene is a primary target of peroxisome proliferator-activated receptors. J. Biol. Chem. 281, 39607-39619 (2006).

76. Colle, R. et al. PPAR-gamma agonists for the treatment of major depression: a review. Pharmacopsychiatry 50, 49-55 (2017).

77. Milanesi, E. et al. Insulin-like growth factor binding protein 2 in bipolar disorder: an expression study in peripheral tissues. World J. Biol. Psychiatry 1-9 (2017).

78. Bezchlibnyk, Y. B., Xu, L., Wang, J. F. \& Young, L. T. Decreased expression of insulin-like growth factor binding protein 2 in the prefrontal cortex of subjects with bipolar disorder and its regulation by lithium treatment. Brain Res. 1147, 213-217 (2007).

79. Guo, M. et al. Role of the adipose PPARgamma-adiponectin axis in susceptibility to stress and depression/anxiety-related behaviors. Mol. Psychiatry 22 1056-1068 (2016) 